\title{
Extremal decomposition of multidimensional complex space for five domains
}

\author{
Yaroslav Zabolotnit, Iryna Denega
}

(Presented by O. A. Dovgoshey)

\begin{abstract}
The paper is devoted to one open extremal problem in geometric function theory of complex variables associated with estimates of functional defined on the systems of non-overlapping domains. We consider problem of the maximum of product of inner radii of $n$ nonoverlapping domains containing points of the unit circle and the power $\gamma$ of the inner radius of domain containing the origin. The problem was formulated in 1994 in the Dubinin paper in the journal "Russian Mathematical Surveys" in the list of unsolved problems and then repeated in his monograph in 2014. Currently it is not solved in general. In this paper we obtained a solution of the problem for five simply connected domains and power $\gamma \in(1 ; 2,57]$ and generalized this result to the case of multidimensional complex space.
\end{abstract}

2010 MSC. 30C75, 32A30.

Key words and phrases. Inner radius of the domain, non-overlapping domains, polycylindrical domain, radial system of points, separating transformation, quadratic differential, Green's function.

Let $\mathbb{N}, \mathbb{R}$ be the sets of natural and real numbers, respectively, $\mathbb{C}$ be the complex plane, $\overline{\mathbb{C}}=\mathbb{C} \bigcup\{\infty\}$ be its one point compactification and $\mathbb{R}^{+}=(0, \infty)$. Let $n \in \mathbb{N}$. A set of points $A_{n}:=\left\{a_{k} \in \mathbb{C}: k=\overline{1, n}\right\}$ is called $n$-radial system if $\left|a_{k}\right| \in \mathbb{R}^{+}, k=\overline{1, n}$, and $0=\arg a_{1}<\arg a_{2}<$ $\ldots<\arg a_{n}<2 \pi$. Let $B$ be the domain in $\overline{\mathbb{C}}$. Let

$$
g_{B}(B, a)=h_{B, a}(z)+\log \frac{1}{|z-a|}
$$

be generalized Green function of the domain $B$ relatively to a point $a \in B$. If $a \rightarrow \infty$ then

$$
g_{B}(B, \infty)=h_{B, \infty}(z)+\log \frac{1}{|z|} .
$$

Received 03.04.2018 
By $r(B, a):=\exp \left(h_{B, a}(z)\right)$ we denote the inner radius of the domain $B \subset \overline{\mathbb{C}}$ with respect to a point $a \in B$ (see, for example, [1-5]). Denote

$$
\begin{aligned}
& \alpha_{k}:=\frac{1}{\pi} \arg \frac{a_{k+1}}{a_{k}}, \quad \alpha_{n+1}:=\alpha_{1}, \quad k=\overline{1, n}, \\
& \sum_{k=1}^{n} \alpha_{k}=2, \quad \Gamma:=\{w \in \mathbb{C}:=|w|=1\} .
\end{aligned}
$$

A finite set of arbitrary domains $\left\{B_{k}\right\}_{k=1}^{n}, n \in \mathbb{N}, n \geq 2$ such as $B_{k} \subset \overline{\mathbb{C}}, B_{k} \cap B_{m}=\emptyset, k \neq m, k, m=\overline{1, n}$, is called a system of nonoverlapping domains.

One of the main concepts in this paper is a notion of quadratic differential. Quadratic differential is a convenient tool for describing extremals on the plane. A great collection of definitions and results about quadratic differentials one can find in monographs $[6,7]$.

The emergence in the theory of univalent functions of extremal problems of non-overlapping domains associated with the paper of M. Lavrentyev, who in 1934 solved the problem of product of conformal radii of two mutually non-overlapping simply connected domains. According to Lavrentyev's result, when $a_{1}$ and $a_{2}$ be finite points then for any pair of non-overlapping simply connected domains $D_{1}$ and $D_{2}$ such that $a_{k} \in D_{k}$, $k=1,2$, the inequality holds

$$
r\left(D_{1}, a_{1}\right) r\left(D_{2}, a_{2}\right) \leq\left|a_{1}-a_{2}\right|^{2},
$$

where equality is attained for the half-planes $D_{k}$ and points $a_{k}$, symmetric with respect to their common boundary. The value

$$
T_{2}:= \begin{cases}r\left(D_{1}, a_{1}\right) r\left(D_{2}, a_{2}\right)\left|a_{1}-a_{2}\right|^{-2}, & a_{1}+a_{2} \neq \infty \\ r\left(D_{1}, a_{1}\right) r\left(D_{2}, a_{2}\right), & a_{1}+a_{2}=\infty,\end{cases}
$$

$a_{k} \in D_{k} \subset \overline{\mathbb{C}}, k=1,2$, is an invariant relative to the fractional-linear mapping of the plane $\overline{\mathbb{C}}$ on itself. That is for any function of the type $f(z)=(a z+b) /(c z+d)$ where $a d-b c \neq 0$ the inequality holds

$$
T_{2}\left(a_{1}, a_{2}, D_{1}, D_{2}\right)=T_{2}\left(f\left(a_{1}\right), f\left(a_{2}\right), f\left(D_{1}\right), f\left(D_{2}\right)\right) .
$$

Thus, the inequality (1) can be considered as upper estimation of the mebius invariant

$$
T_{2} \leq 1
$$


In the case of three or more points many authors [3-5] considered estimations of a more general mebius invariant of the form

$$
T_{n}:=\frac{\prod_{k=1}^{n} r\left(D_{k}, a_{k}\right)}{\left\{\prod_{1 \leqslant k<l \leqslant n}^{\prime}\left|a_{k}-a_{l}\right|\right\}^{\frac{2}{n-1}}},
$$

(here the stroke in the product means that for an infinitely distant point under the corresponding factor we mean the unit). In order to get $T_{n}$ it is necessary for $n$ pairwise non-overlapping domains $D_{k}$ and points $a_{k} \in D_{k}, k=\overline{1, n}, n \geq 2$, multiply all possible pairwise products of type (2) and take the root of degree $2(n-1)$.

In 1951 G.Goluzin summarized the Lavrentyev problem in the case of a finite number of $n, n \geqslant 3$ mutually non-overlapping simply connected domains $B_{k}, a_{k} \in B_{k} \subset \mathbb{C}\left(\left\{a_{k}\right\}_{k=1}^{n}\right.$ are arbitrary fixed finite and various points of the complex plane), and at $n=3$ obtained an exact estimate for $T_{3}[2$, p. 165]

$$
T_{3} \leq \frac{64}{81 \sqrt{3}}
$$

where equality is achieved, with the precision of fractional-linear transformations, only for domains that are equal angles and points lying on the bisectors of these angles at identical distances from their common vertex.

The case $n>3$ is more complicated and significantly different from the situation $n \leqslant 3$, since by the fractional-linear mapping any three predefined points can be converted into three points that are convenient to prove.

For $n=4$ G. Kuz'mina [3, p. 25] showed that the problem of estimating $T_{4}$ reduces to the smallest capacity problems in a certain family of continuums and obtained the exact inequality

$$
T_{4} \leq 3^{2} \cdot 4^{-8 / 3}
$$

Equality is achieved only in the case when with precision to fractionallinear transformation $a_{1}=-a_{2}=1, a_{3}=-a_{4}=i(2-\sqrt{3})$, and the boundary of the set $\bigcup_{k=1}^{4} D_{k}$ consists with segments $[\sqrt{3}-2,2-\sqrt{3}]$, rays $\{z \mid z=i t, t \in[1,+\infty)\}$ and $\{z \mid z=i t, t \in[-\infty,-1)\}$, part of the circular arc $\rho$, passing through points $-1, i, 2-\sqrt{3}$, which lies in the 
first quadrant, and circular arcs that we get from $\rho$ by transformations $w \rightarrow-w, w \rightarrow \bar{w}, w \rightarrow-\bar{w}$.

For $n \geq 5$ full solution (3) is not obtained at this time. Since, the evaluation of the product of conformal radii of mutually non-overlapping domains if $n \geq 5$ without any restriction on the domains $B_{k}$ and points $a_{k}, k=1, \ldots, 5$ is quite difficult and interesting problem.

In $1975 \mathrm{~N}$. Lebedev in the monograph [8, p. 32-33] considered the more general extremal problem on product of conformal radii.

Problem 1. There are $n$ various fixed points $a_{k}, k=\overline{1, n}, n>3$, on a plane $w$. Functions $w=f_{k}(z), k=\overline{1, n}$, are regular in the circle $|z|<1$ and univalent map circle $|z|<1$ onto non-overlapping domains $B_{k}$, which contain the corresponding points $a_{k}, k=\overline{1, n}$, and in such a way, that $f_{k}(0)=a_{k}, k=\overline{1, n}$. What about maximum of product

$$
\prod_{k=1}^{n}\left|f_{k}^{\prime}(0)\right|^{\gamma_{k}} \longrightarrow \max , \quad \gamma_{k}>0, n>3
$$

relatively to any functions $f_{k}(z), k=\overline{1, n}$ ?

However, this problem is generally not solved so far. Further this problem was generalized to more general classes of multiply connected domains replacing conformal radius to the inner radius.

Consider an extremal problem which was formulated in 1994 in the works of $\mathrm{V}$. Dubinin $[4,9]$ in the lists of unsolved problems.

Problem 2. [4, p. 68]. Show that for pairwise disjoint domains $B_{0}$, $B_{1}, B_{2}, \ldots, B_{n}, n \geq 2$, in $\overline{\mathbb{C}}$, system of points $A_{n}=\left\{a_{k}\right\}_{k=1}^{n}, a_{0}=0$, $\left|a_{k}\right|=1, k=\overline{1, n}$, and number $\gamma \leq n$, maximum of the product

$$
I_{n}(\gamma)=r^{\gamma}\left(B_{0}, 0\right) \prod_{k=1}^{n} r\left(B_{k}, a_{k}\right),
$$

is attained for configuration of domains $B_{k}$ and points $a_{k}$ possessing rotational $n$-symmetry.

Currently it is not solved in general only partial results are known. In 1988 in [10] this problem was solved for $\gamma=1$ and $n \geq 2$, and from the method of this work it implies that the result is true and for $0<\gamma<1$. In 1996 Kovalev [11] got the solution to this problem with some restrictions on the geometry location of sets of points on the unit circle and, namely, for $n \geq 5$ and subclass points systems satisfying condition

$$
0<\alpha_{k} \leq 2 / \sqrt{\gamma}, k=\overline{1, n}
$$


It is clear that these conditions are sufficiently stringent conditions, significantly narrowing the set of feasible configurations. It should be noted that the Kovalev result is interesting not only by itself, but the method of study is important too. In 2003 in the paper of G. Kuz'mina [12] in the case of simply connected domains this problem has also been studied for $\gamma \in(0,1]$ by another method. In 2008 Bakhtin [5, p. 255] complemented the ideas and methods of previous works and thus he showed that the result of $\mathrm{V}$. Dubinin holds for an arbitrary $\gamma \in(0, \infty)$ but since some number $n_{0}(\gamma)$. It should be noted in the case $\gamma>1$ method developed in the paper of V. Dubinin [10] can not be applied. In papers [13-18] particular cases of the Dubinin problem were considered. Note that the cases $n=2,3,4$ with $\gamma>1$ are the most difficult cases in this issue.

It is well known (see, for example, [19-21]) that

$$
\mathbb{C}^{l}=\underbrace{\mathbb{C} \times \mathbb{C} \times \mathbb{C} \times \ldots \times \mathbb{C}}_{l \text {-times }}, \quad l \in \mathbb{N} .
$$

Let $\overline{\mathbb{C}}^{l}=\underbrace{\overline{\mathbb{C}} \times \overline{\mathbb{C}} \times \overline{\mathbb{C}} \times \ldots \times \overline{\mathbb{C}}}_{l \text {-times }}$ be compactification of the space $\mathbb{C}^{l}$ where the set of infinitely distant points has complex dimension $l-1$ (see, for example, [19-21]). And let $\Omega\left(\omega_{1}, \omega_{2}, \ldots, \omega_{l}\right)$ be a point of the space $\mathbb{C}^{l}$ with coordinates $\omega_{k}, k=\overline{1, l}$. By $[D]^{l}$ (cartesian degree of the domain $D \in \overline{\mathbb{C}})$ we denote the cartesian product $\underbrace{D \times D \times \ldots \times D}_{l \text {-times }}$. Besides by $[d]^{l}$ (cartesian degree of a point $d \in \overline{\mathbb{C}}$ ) we denote the point in $\overline{\mathbb{C}}^{l}$ which has coordinates $\underbrace{(d, \ldots, d)}_{l-\text { times }}$.

A domain $\mathbb{B}=\mathbf{B}_{1} \times \mathbf{B}_{2} \times \mathbf{B}_{3} \times \ldots \times \mathbf{B}_{l}$ is called polycylindrical domain in $\overline{\mathbb{C}}^{l}$ (see [19]), where the domains $\mathbf{B}_{k} \subset \overline{\mathbb{C}}, k=\overline{1, l}$, and $\mathbf{B}_{k}$ are called coordinate domains.

A system $\left\{\mathbb{B}_{k}\right\}_{k=1}^{l}\left(\mathbb{B}_{k}=B_{1}^{(k)} \times \ldots \times B_{l}^{(k)}, k=\overline{1, l}\right)$ is called a system of polycylindrical non-overlapping domains if for each fixed $p_{0}, p_{0}=$ $\overline{1, l}$, the system of domains $\left\{B_{p_{0}}^{(k)}\right\}, k=\overline{1, l}$, is the system of nonoverlapping domains in $\overline{\mathbb{C}}$.

A value

$$
\mathbb{R}(\mathbb{B}, \Omega)=\left[\prod_{k=1}^{l} r\left(\mathbf{B}_{k}, \omega_{k}\right)\right]^{\frac{1}{l}}
$$

is called generalized inner radius of the polycylindrical domain $\mathbb{B}$ in point $\Omega(\Omega \in \mathbb{B})$. If $l=1$ then the value $\mathbb{R}(\mathbb{B}, \Omega)$ is usual inner radius of the domain $\mathbb{B} \subset \overline{\mathbb{C}}$ relatively to a point $\Omega$. 
In the paper we consider the Problem 2 for five pairwise disjoint simply connected domains in the complex plane. Currently the theorem 1 of the paper [15] is the best result for $n=4$ without any additional conditions on the location of the points $a_{k}, k=\overline{1,4}$, where the Dubinin problem was solved for $\gamma \in(1 ; 2,09]$. In the paper the following result is obtained.

Theorem 1. Let $\mathbb{B}_{k}=\mathbf{B}_{1}^{(k)} \times \mathbf{B}_{2}^{(k)} \times \mathbf{B}_{3}^{(k)} \times \ldots \times \mathbf{B}_{l}^{(k)}, k=\overline{0,4}$, is a system of mutually non-overlapping polycylindrical domains in $\mathbb{C}^{l}$ and points $\Omega_{k}=\left(\omega_{1}^{(k)}, \omega_{2}^{(k)}, \ldots, \omega_{l}^{(k)}\right), k=\overline{0,4}$, satisfy the following conditions $\Omega_{0}=(0,0, \ldots, 0), \Omega_{k} \in \mathbb{B}_{k}$ and for any $m=\overline{1, l}, \mathbf{B}_{m}^{0}, \mathbf{B}_{m}^{1}, \mathbf{B}_{m}^{2}, \mathbf{B}_{m}^{3}, \mathbf{B}_{m}^{4}$ are pairwise disjoint simply connected domains in $\overline{\mathbb{C}},\left|\omega_{m}^{k}\right|=1, k=\overline{1,4}$, and $\gamma \in(1 ; 2,57]$. Then the following inequality holds

$$
\mathbb{R}^{\gamma}\left(\mathbb{B}_{0}, \Omega_{0}\right) \prod_{k=1}^{4} \mathbb{R}\left(\mathbb{B}_{k}, \Omega_{k}\right) \leq \frac{\left(\frac{\gamma}{4}\right)^{\frac{\gamma}{4}}}{\left(1-\frac{\gamma}{16}\right)^{4+\frac{\gamma}{4}}}\left(\frac{1-\frac{\sqrt{\gamma}}{4}}{1+\frac{\sqrt{\gamma}}{4}}\right)^{2 \sqrt{\gamma}}
$$

One of the extremal system is the system

$$
\begin{gathered}
\left\{\mathbb{B}_{k}\right\}_{k=0}^{4}=\left\{\left[B_{0}^{(0)}\right]^{l},\left[B_{1}^{(0)}\right]^{l},\left[B_{2}^{(0)}\right]^{l},\left[B_{3}^{(0)}\right]^{l},\left[B_{4}^{(0)}\right]^{l}\right\}, \\
\left\{\Omega_{k}\right\}_{k=0}^{4}=\left\{[0]^{l},\left[\omega_{1}^{(0)}\right]^{l},\left[\omega_{2}^{(0)}\right]^{l},\left[\omega_{3}^{(0)}\right]^{l},\left[\omega_{4}^{(0)}\right]^{l}\right\},
\end{gathered}
$$

where domains $B_{k}^{(0)}$ and points $\omega_{k}^{(0)}, k=\overline{0,4}$, are, respectively, circular domains and poles of the quadratic differential

$$
G(w) d w^{2}=-\frac{(16-\gamma) w^{4}+\gamma}{w^{2}\left(w^{4}-1\right)^{2}} d w^{2} .
$$

Proof. Perform the following transformations

$$
\begin{gathered}
\mathbb{R}^{\gamma}\left(\mathbb{B}_{0}, \Omega_{0}\right) \cdot \prod_{k=1}^{4} \mathbb{R}\left(\mathbb{B}_{k}, \Omega_{k}\right)=\left[\prod_{m=1}^{l} r\left(\mathbf{B}_{m}^{(0)}, \Omega_{m}^{(0)}\right)\right]^{\frac{\gamma}{l}} . \\
\prod_{k=1}^{4}\left[\prod_{m=1}^{l} r\left(\mathbf{B}_{m}^{(k)}, \Omega_{m}^{(k)}\right)\right]^{\frac{1}{l}}=\left[\prod_{m=1}^{l}\left[\left(r\left(\mathbf{B}_{m}^{(0)}, \Omega_{m}^{(0)}\right)\right)^{\gamma} \prod_{k=1}^{4} r\left(\mathbf{B}_{m}^{(k)}, \Omega_{m}^{(k)}\right)\right]\right]^{\frac{1}{l}} .
\end{gathered}
$$

Then for $\forall m=\overline{1, l}$ the domains $\mathbf{B}_{m}^{(k)}, k=\overline{0, n}$, make up the system of pairwise disjoint simply connected domains in the complex plane $\overline{\mathbb{C}}$.

Thus, prove the following lemma. 
Lemma 1. Let $n=4, \gamma \in(1 ; 2,57]$. Then the following sharp estimate holds

$$
r^{\gamma}\left(B_{0}, 0\right) \prod_{k=1}^{4} r\left(B_{k}, a_{k}\right) \leq \frac{\left(\frac{\gamma}{4}\right)^{\frac{\gamma}{4}}}{\left(1-\frac{\gamma}{16}\right)^{4+\frac{\gamma}{4}}}\left(\frac{1-\frac{\sqrt{\gamma}}{4}}{1+\frac{\sqrt{\gamma}}{4}}\right)^{2 \sqrt{\gamma}}
$$

where $B_{0}, B_{1}, B_{2}, B_{3}, B_{4}$ are pairwise disjoint simply connected domains in $\overline{\mathbb{C}}, a_{0}=0,\left|a_{k}\right|=1, k=\overline{1,4}, r\left(B_{j}, a_{j}\right)$ is the inner radius of the domain $B_{j}$ with respect to the point $a_{j}\left(a_{j} \in B_{j}\right), j=\overline{0,4}$. Equality is attained if $d_{k}$ and $D_{k}, k=\overline{0, n}$, are, respectively, poles and circular domains of the quadratic differential (4).

Proof. Find the value

$$
I_{n}^{0}(\gamma)=r^{\gamma}\left(D_{0}, 0\right) \prod_{k=1}^{n} r\left(D_{k}, d_{k}\right)
$$

where $0 \cup\left\{d_{k}\right\}_{k=1}^{n}$ and $\left\{D_{k}\right\}_{k=0}^{n}$ are, respectively, poles and circular domains of the quadratic differential (4). From results of the papers $[4,5,11]$ and properties of the separating transformation we have

$$
I_{n}^{0}(\gamma)=\left(\frac{2}{n}\right)^{n}\left(\frac{2^{\frac{4 \gamma}{n^{2}}+6}\left(\frac{2 \sqrt{\gamma}}{n}\right)^{\frac{4 \gamma}{n^{2}}}}{\left(2-\frac{2 \sqrt{\gamma}}{n}\right)^{\frac{1}{2}\left(2-\frac{2 \sqrt{\gamma}}{n}\right)^{2}}\left(2+\frac{2 \sqrt{\gamma}}{n}\right)^{\frac{1}{2}\left(2+\frac{2 \sqrt{\gamma}}{n}\right)^{2}}}\right)^{\frac{n}{2}}
$$

Using elementary calculations we obtain that the following estimate holds

$$
I_{n}^{0}(\gamma)=\left(\frac{4}{n}\right)^{n} \frac{\left(\frac{4 \gamma}{n^{2}}\right)^{\frac{\gamma}{n}}}{\left(1-\frac{\gamma}{n^{2}}\right)^{n+\frac{\gamma}{n}}}\left(\frac{1-\frac{\sqrt{\gamma}}{n}}{1+\frac{\sqrt{\gamma}}{n}}\right)^{2 \sqrt{\gamma}}
$$

Thus, for $n=4$ we have

$$
I_{4}^{0}(\gamma)=\frac{\left(\frac{\gamma}{4}\right)^{\frac{\gamma}{4}}}{\left(1-\frac{\gamma}{16}\right)^{4+\frac{\gamma}{4}}}\left(\frac{1-\frac{\sqrt{\gamma}}{4}}{1+\frac{\sqrt{\gamma}}{4}}\right)^{2 \sqrt{\gamma}}
$$

The following statement holds.

Lemma 2. [18] Let $n \in \mathbb{N}, n \geq 2, \gamma>0$. And let $\left\{B_{0}, B_{1}, B_{2}, \ldots, B_{n}\right\}$ be the system of pairwise disjoint simply connected domains such that $0 \in B_{0}, a_{k} \in B_{k} \cap \Gamma, k=\overline{1, n}$, and $r^{\gamma}\left(B_{0}, 0\right) \prod_{k=1}^{n} r\left(B_{k}, a_{k}\right)>I_{n}^{0}(\gamma)$.

Then we have inequality

$$
r\left(B_{0}, 0\right) \leq n^{-\frac{n}{2(n-\gamma)}} I_{n}^{0}(\gamma)^{-\frac{1}{n-\gamma}} .
$$


Taking lemma 2 and result of the paper [13] into account we consider the case

$$
r\left(B_{0}, 0\right) \leq 4^{-\frac{2}{(4-\gamma)}} I_{4}^{0}(\gamma)^{-\frac{1}{4-\gamma}}, \quad \alpha_{0}>\frac{2}{\sqrt{\gamma}}, \quad \alpha_{0}=\max _{k} \alpha_{k} .
$$

We will prove that under the given conditions the following inequality holds

$$
\frac{r^{\gamma}\left(B_{0}, 0\right) \prod_{k=1}^{4} r\left(B_{k}, a_{k}\right)}{\frac{\left(\frac{\gamma}{4}\right)^{\frac{\gamma}{4}}}{\left(1-\frac{\gamma}{16}\right)^{4+\frac{\gamma}{4}}}\left(\frac{1-\frac{\sqrt{\gamma}}{4}}{1+\frac{\sqrt{\gamma}}{4}}\right)^{2 \sqrt{\gamma}}}<1
$$

From Lemma 2

$$
r^{\gamma}\left(B_{0}, 0\right)<4^{-\frac{2 \gamma}{(4-\gamma)}} I_{4}^{0}(\gamma)^{-\frac{\gamma}{4-\gamma}}
$$

Further, using Theorem 1 of the paper [3] the following inequality holds

$$
\begin{gathered}
\prod_{k=1}^{4} r\left(B_{k}, a_{k}\right) \\
\leq \frac{9}{4^{\frac{8}{3}}}\left(\left|a_{1}-a_{2}\right| \cdot\left|a_{1}-a_{3}\right| \cdot\left|a_{2}-a_{3}\right| \cdot\left|a_{1}-a_{4}\right| \cdot\left|a_{2}-a_{4}\right| \cdot\left|a_{3}-a_{4}\right|\right)^{\frac{2}{3}} .
\end{gathered}
$$

Let $\alpha_{0}=\alpha_{4}$ then we obtain

$$
\begin{gathered}
\prod_{k=1}^{4} r\left(B_{k}, a_{k}\right) \leq \frac{144}{4^{\frac{8}{3}}}\left(\sin \frac{\pi \alpha_{1}}{2} \sin \frac{\pi \alpha_{2}}{2} \sin \frac{\pi \alpha_{3}}{2}\right)^{\frac{2}{3}} \\
\times\left(\sin \frac{\pi\left(\alpha_{1}+\alpha_{2}\right)}{2} \sin \frac{\pi\left(\alpha_{2}+\alpha_{3}\right)}{2} \sin \frac{\pi\left(\alpha_{1}+\alpha_{2}+\alpha_{3}\right)}{2}\right)^{\frac{2}{3}} .
\end{gathered}
$$

If $\alpha_{0}>\frac{2}{\sqrt{\gamma}}$ then $\alpha_{1}+\alpha_{2}+\alpha_{3}<2-\frac{2}{\sqrt{\gamma}}$. Consider the right side of the expression (6) under these conditions. The following inequality holds

$$
\begin{gathered}
\prod_{k=1}^{4} r\left(B_{k}, a_{k}\right) \\
\leq \frac{144}{4^{\frac{8}{3}}} \sin ^{2}\left(2-\frac{2}{\sqrt{\gamma}}\right)\left(\frac{\pi}{6}\right) \sin ^{\frac{4}{3}}\left(2-\frac{2}{\sqrt{\gamma}}\right)\left(\frac{\pi}{3}\right) \sin ^{\frac{2}{3}}\left(2-\frac{2}{\sqrt{\gamma}}\right)\left(\frac{\pi}{2}\right) .
\end{gathered}
$$

Thus,

$$
\frac{r^{\gamma}\left(B_{0}, 0\right) \prod_{k=1}^{4} r\left(B_{k}, a_{k}\right)}{\frac{\left(\frac{\gamma}{4}\right)^{\frac{\gamma}{4}}}{\left(1-\frac{\gamma}{16}\right)^{4+\frac{\gamma}{4}}}\left(\frac{1-\frac{\sqrt{\gamma}}{4}}{1+\frac{\sqrt{\gamma}}{4}}\right)^{2 \sqrt{\gamma}}}
$$




$$
\leq \frac{144 \sin ^{2}\left(2-\frac{2}{\sqrt{\gamma}}\right)\left(\frac{\pi}{6}\right) \sin ^{\frac{4}{3}}\left(2-\frac{2}{\sqrt{\gamma}}\right)\left(\frac{\pi}{3}\right) \sin ^{\frac{2}{3}}\left(2-\frac{2}{\sqrt{\gamma}}\right)\left(\frac{\pi}{2}\right)}{4^{\frac{8}{3}+\frac{2 \gamma}{(4-\gamma)}}\left(I_{4}^{0}(\gamma)\right)^{\frac{4}{4-\gamma}}}:=Q(\gamma) .
$$

Substituting in the right side of the last inequality the value $\gamma=2,57$, we obtain

$$
\frac{r^{\gamma}\left(B_{0}, 0\right) \prod_{k=1}^{4} r\left(B_{k}, a_{k}\right)}{\frac{\left(\frac{\gamma}{4}\right)^{\frac{\gamma}{4}}}{\left(1-\frac{\gamma}{16}\right)^{4+\frac{\gamma}{4}}}\left(\frac{1-\frac{\sqrt{\gamma}}{4}}{1+\frac{\sqrt{\gamma}}{4}}\right)^{2 \sqrt{\gamma}}} \leq 1 .
$$

Therefore for $\gamma=2,57$ the inequality (5) is valid. Let now $\gamma<2,57$. Prove that the function $Q(\gamma)$ monotonically increases for $1<\gamma \leq 2,57$. It is clear that the functions $\sin \left(2-\frac{2}{\sqrt{\gamma}}\right)\left(\frac{\pi}{6}\right), \sin \left(2-\frac{2}{\sqrt{\gamma}}\right)\left(\frac{\pi}{3}\right)$, $\sin \left(2-\frac{2}{\sqrt{\gamma}}\right)\left(\frac{\pi}{2}\right)$ are increasing on this interval. And thus the numerator of the expression for the function $Q(\gamma)$ is increasing. Further, consider the denominator of the expression $Q(\gamma)$. So,

$$
4^{\frac{8}{3}+\frac{2 \gamma}{(4-\gamma)}}\left(I_{4}^{0}(\gamma)\right)^{\frac{4}{4-\gamma}}=4^{\frac{8}{3}}\left(2^{\gamma}\left(I_{4}^{0}(\gamma)\right)\right)^{\frac{4}{4-\gamma}}
$$

Prove that the expression

$$
\left(2^{\gamma}\left(I_{4}^{0}(\gamma)\right)\right)^{\frac{4}{4-\gamma}}=\left(2^{\gamma} \frac{\left(\frac{\gamma}{4}\right)^{\frac{\gamma}{4}}}{\left(1-\frac{\gamma}{16}\right)^{4+\frac{\gamma}{4}}}\left(\frac{1-\frac{\sqrt{\gamma}}{4}}{1+\frac{\sqrt{\gamma}}{4}}\right)^{2 \sqrt{\gamma}}\right)^{\frac{4}{4-\gamma}}
$$

monotonically decreases for $1<\gamma \leq 2,57$. Taking logarithmic derivative of the last expression we have that for $1<\gamma \leq 2,57$ it is negative and it means that denominator of the expression $Q(\gamma)$ decreases. Summarizing all of the above we obtain that the expression $Q(\gamma)$ increases on the interval $1<\gamma \leq 2,57$ and therefore for any $1<\gamma<2,57$ the following inequality holds

$$
Q(\gamma)<Q(2,57)<1
$$

Hence, for any $\gamma$ such that $1<\gamma<2,57$ and for any configuration of domains $B_{k}$ and points $a_{k}, k=\overline{0,4}$, satisfying all conditions of the Lemma 1, the inequality (5) holds. Lemma 1 is proved.

Therefore, we obtain the inequality of the Theorem 1

$$
\mathbb{R}^{\gamma}\left(\mathbb{B}_{0}, \Omega_{0}\right) \prod_{k=1}^{4} \mathbb{R}\left(\mathbb{B}_{k}, \Omega_{k}\right) \leq\left[\prod_{m=1}^{l}\left[\frac{\left(\frac{\gamma}{4}\right)^{\frac{\gamma}{4}}}{\left(1-\frac{\gamma}{16}\right)^{4+\frac{\gamma}{4}}}\left(\frac{1-\frac{\sqrt{\gamma}}{4}}{1+\frac{\sqrt{\gamma}}{4}}\right)^{2 \sqrt{\gamma}}\right]\right]^{\frac{1}{l}}
$$




$$
=\frac{\left(\frac{\gamma}{4}\right)^{\frac{\gamma}{4}}}{\left(1-\frac{\gamma}{16}\right)^{4+\frac{\gamma}{4}}}\left(\frac{1-\frac{\sqrt{\gamma}}{4}}{1+\frac{\sqrt{\gamma}}{4}}\right)^{2 \sqrt{\gamma}}
$$

Theorem 1 is proved.

\section{References}

[1] M. A. Lavrent'ev, On the theory of conformal mappings // Tr. Sci. Inst An USSR, 5 (1934), 159-245 (in Russian).

[2] G. M. Goluzin, Geometric theory of functions of a complex variable, Amer. Math. Soc. Providence, R.I. 1969.

[3] G. V. Kuz'mina, Methods of geometric function theory II // St. Petersbg. Math. J., 9 (1998), No. 5, 889-930.

[4] V. N. Dubinin, Symmetrization method in geometric function theory of complex variables // Successes Mat. Science, 49 (1994), No. 1, 3-76 (in Russian); translation in Russian Math. Surveys, 1 (1994), No. 1, 1-79.

[5] A. K. Bakhtin, G. P. Bakhtina, Yu. B. Zelinskii, Topological-algebraic structures and geometric methods in complex analysis // Zb. prats of the Inst. of Math. of NASU, 2008 (in Russian).

[6] J. Jenkins, Univalent functions and conformal mapping, Moscow:Publishing House of Foreign Literature, 256 (1962) (in Russian).

[7] K. Strebel, Quadratic differentials, Springer-Verlag, 1984.

[8] N. A. Lebedev, The area principle in the theory of univalent functions, Moscow, Science, 1975 (in Russian).

[9] V. N. Dubinin, Condenser capacities and symmetrization in geometric function theory, Birkhäuser/Springer, Basel, 2014.

[10] V. N. Dubinin, Separating transformation of domains and problems on extremal decomposition // Notes scientific. sem. Leningr. Dep. of Math. Inst. AN USSR., 168 (1988), 48-66 (in Russian); translation in J. Soviet Math., 53 (1991), No. 3, $252-263$.

[11] L. V. Kovalev, On the problem of extremal decomposition with free poles on a circle // Dal'nevostochnyi Mat. Sb., (1996), No. 2, 96-98 (in Russian).

[12] G. V. Kuzmina, Extremal metric method in problems of the maximum of product of powers of conformal radii of non-overlapping domains with free parameters // Notes scientific. sem. Leningr. Dep. of Math. Inst. AN USSR., 302 (2003), 52-67 (in Russian); translation in Journal of Mathematical Sciences (New York), 129 (2005), No. 3, 3843-3851.

[13] A. K. Bakhtin, I. V. Denega, Addendum to a theorem on extremal decomposition of the complex plane // Bulletin de la société des sciences et des lettres de Eódź, Recherches sur les déformations, 62 (2012), No. 2, 83-92. 
[14] Ja. V. Zabolotnij, Determination of the maximum of a product of inner radii of pairwise nonoverlapping domains // Dopov. Nac. akad. nauk Ukr., (2016), No. 3, 7-13 (in Ukrainian).

[15] A. K. Bakhtin, I. Ya. Dvorak, Ya. V. Zabolotnyi, Estimates of the product of inner radii of five nonoverlapping domains // Ukr. Mat. Zh., 69 (2017), No. 2, 261-267.

[16] I. V. Denega, Ya. V. Zabolotnii, Estimates of products of inner radii of nonoverlapping domains in the complex plane // Complex Variables and Elliptic Equations, 62 (2017), No. 11, 1611-1618.

[17] A. Bakhtin, L. Vygivska, I. Denega, N-radial systems of points and problems for non-overlapping domains // Lobachevskii Journal of Mathematics, 38 (2017), No. 2, 229-235.

[18] A. K. Bakhtin, Estimates of inner radii for mutually disjoint domains // Zb. prats of the Inst. of Math. of NASU, 14 (2017), No. 1, 1-9 (in Russian).

[19] B. V. Shabat, Introduction to complex analysis. Part II. Functions of several variables, Second edition, revised and augmented. Izdat. "Nauka", Moscow, 1976 (in Russian).

[20] E. M. Chirka, Complex analytic sets, Nauka, Moscow, 1985 (in Russian).

[21] B. A. Fuks, Introduction to the theory of analytic functions of several complex variables. Gosudarstv, Izdat. Fiz.-Mat. Lit., Moscow, 1962 (in Russian).

\section{CONTACT INFORMATION}

Yaroslav Zabolotnii Institute of mathematics of National

Academy of Sciences of Ukraine,

Kyiv, Ukraine

E-Mail: yaroslavzabolotnii@gmail.com

\section{Iryna Denega}

Institute of mathematics of National

Academy of Sciences of Ukraine,

Kyiv, Ukraine

E-Mail: iradenega@gmail.com 